\title{
Determining Internal Local Corrosion of Screwed Pipes Through Ultrasonic Testing
}

\author{
Toshio Fukuda, ${ }^{2}$ OShintaro Sakamoto, ${ }^{1}$ Fumihito Arai, $*^{2}$ Yasuhisa Hasegawa,*² \\ Futoshi Kobayashi, ${ }^{* 3}$ Hironori Yui, ${ }^{2}$ and Yasunori Abe ${ }^{* 1}$
}

\author{
*l Shinryo Corporation, Research and Development Center \\ 41, Wadai, Tsukuba, Ibaraki 300-42, Japan \\ *2 Nagoya University, Dept. of Micro System Engineering, Dept. of Mechano-Informatics and Systems, \\ Fro-cho, Chikusa-ku, Nagoya, 464-8603, Japan \\ *3 Kobe University, Dept. of System Function Science, \\ 1-1, Rokkodai-cho, Nada-ku, Kobe, 657-8501, Japan \\ sakamoto.sh@shinryo.com
}

\begin{abstract}
In diagnosing air-conditioning pipe deterioration, it is especially important to inspect screwed parts because their original thickness is thinner than that of straight parts and it is comparatively easy for leakage to occur if there is local internal corrosion. Usually, a radiographic test is used, but this method requires a licensed person to carry out the inspection. We examined a method of applying an ultrasonic test for the inspection, and proposed a method for detecting internal local corrosions of screwed parts and estimating the size of corrosion with surface $\mathrm{SH}$ waves.
\end{abstract}

In this paper, we propose a method for detecting internal local corrosion of screwed parts of air-conditioning pipes with ultrasonic testing. The objective of the first step is to detect artificial corrosion and estimate its size and position. It is impossible to detect corrosion with a normal probe because a joint or valve overlapping the screwed pipe prevents ultrasonic waves from reaching the inside. Therefore, we used an angle probe, specifically a surface $\mathrm{SH}$ wave angle probe, because the thickness of the pipes requires a large angle of incidence, close to a right angle. We describe the following in this paper:

- The results of experimental analysis on the echo from screwed parts of pipes.

- The method used to detect local corrosion and estimate the size and position by using the pipe-end echo.

- The results of tests on artificial corrosion pipes and the limitations of this method.

KEYWORDS: Surface SH Wave, Screwed Part of Pipes, Ultrasonic Test, Estimation of Corrosion States

\section{INTRODUCTION}

Pipe deterioration that accompanies aging of air-conditioning equipment causes many problems in buildings, particularly water leakage due to internal pipe corrosion. Therefore, it is very important to inspect for internal corrosion of pipes. Many automatic inspection systems using non-destructive testing have been developed in order to inspect quantitatively without involving difficult work or shutting down plant operations. ${ }^{[1][2]}$ We have also been developing a pipe inspection robot with ultrasonic testing and an ultrasonic data analyzing method. ${ }^{[3][4]}$

It is especially important to inspect the screwed parts of a steel pipe because it is comparatively easy for leakage to occur if there is internal local corrosions.

Radiographic testing is generally used to inspect the parts, but this method requires that a licensed person conduct the inspection. Therefore, we attempted to develop a technique using an ultrasonic angle probe.

Although there are some studies on inspecting screwed parts by ultrasonic testing, ${ }^{[5][6]}$ there have no such studies regarding pipes. We used a surface $\mathrm{SH}$ wave angle probe because the thinness of the 
pipes requires a large angle of incidence ${ }^{[7]}$. In this report, we show the results of analysis of the surface $\mathrm{SH}$ echo from the screwed parts, the method used to detect local corrosion and estimate its size using the echo from the end of the pipe (hereinafter pipe-end echo), and the method used to estimate its position using a simple numerical model. We show the usefulness and the limitations of this method experimentally using artificial corrosion pipes.

\section{ANALYSIS RESULTS OF ECHOES}

\subsection{Surface SH Echo}

Ultrasonic pulse waves cannot spread to the male screwed pipe if a normal incidence probe is used from outside of the pipe joint, as shown in figure 1, due to sealant and an air-layer that differs in the amount of acoustic impedance in male and female screwed parts. Therefore, we considered a method using an angle probe. We decided to use a surface SH probe because the thinness of air-conditioning pipes requires a large angle of incidence, close to a right angle. We used a probe with a ceramic oscillator $10 \times 5 \mathrm{~mm}$ and $5 \mathrm{MHz}$ frequency. The range of direction is shown in figure 2 .

Figure 3 and 4 show examples of the echoes that were measured by the probe. Measurements were taken at several points of a pipe $60.5 \mathrm{~mm}$ in diameter, and the distance from the screw was fixed. In these figures, the echoes from 0 to 10 $\mu \mathrm{sec}$ are transmitted wave and surface echoes, and the echoes from the screwed part appear at about 27 $\mu \mathrm{sec}$. The peak at about $47 \mu \mathrm{sec}$ is the pipe-end echo. In spite of no internal corrosions, the echoes differ at each position.

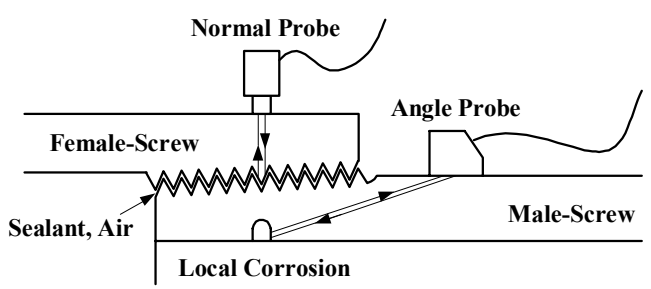

Figure 1 Ultrasonic Inspection of Screwed Pipe

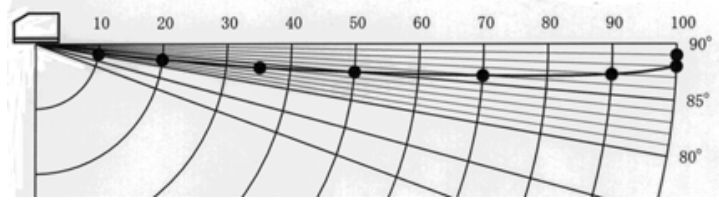

Figure 2 Range of Direction for Surface SH Probe

\subsection{Measuring Conditions}

We experimentally investigated the reason why echoes from the screw differ depending on the circumferential position of the pipe. In the following experiments, the diameter of the pipe is a uniform $60.5 \mathrm{~mm}$, the distance between the tip and the incident point of the probe is $8 \mathrm{~mm}$, and the distance between the tip of the probe and the near edge of the screw is a constant $24 \mathrm{~mm}$. The velocity of the $\mathrm{SH}$ wave is approximately $3,230 \mathrm{~m} / \mathrm{s}$ in steel (see figure 5).

The objective of this inspection is to detect internal corrosion, so it is desirable to reduce the influences of SH waves propagating near the surface of the pipe. Therefore, we applied couplant to the surface of the pipe from the probe tip to the screw edge. ${ }^{[8]}$

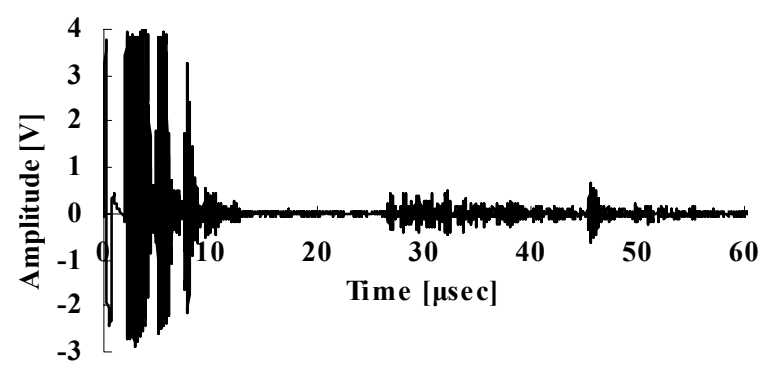

Figure 3 Wave Form of Normal Point (No. 1)

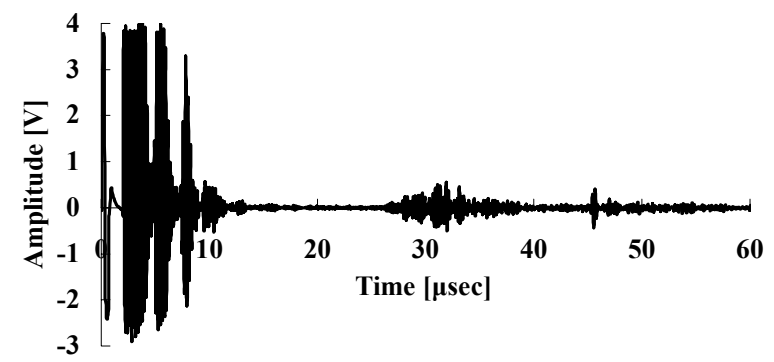

Figure 4 Wave Form of Normal Point (No. 2)

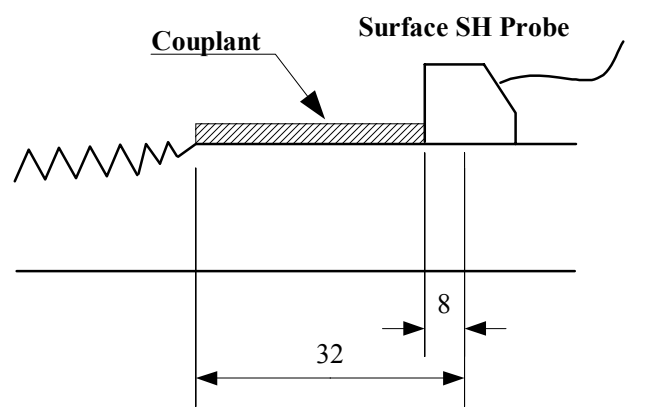

Figure 5 Measurement Position 
Table 1 Surface Effect

\begin{tabular}{|c|c|c|c|c|}
\hline & $\begin{array}{c}\text { Maximum } \\
\text { Amplitude }\end{array}$ & $\begin{array}{c}\text { Minimum } \\
\text { Amplitude }\end{array}$ & $\begin{array}{c}\text { Average } \\
\text { Amplitude }\end{array}$ & $\begin{array}{c}\text { Standard } \\
\text { Deviation }\end{array}$ \\
\hline A & 2.77 & 2.55 & 2.64 & 0.10 \\
\hline B & 2.83 & 2.05 & 2.46 & 0.46 \\
\hline
\end{tabular}

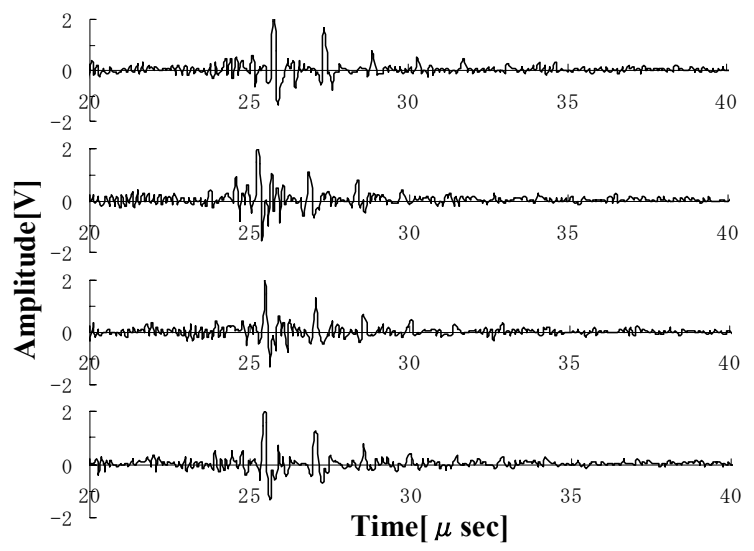

Figure 6 Wave Form on (a)

\subsection{Experimental Analysis}

First, we observed pipe-end echoes by placing the probe on two non-screwed steel pipes (labelled "A" and "B"), and measuring their amplitude. A was made of round steel with boring and both surfaces (interior and exterior) were finished to $6.3 \mathrm{~s}$. B was a standardized carbon steel pipe and only the exterior was finished to $6.3 \mathrm{~s}$. We observed the echoes at five points on each pipe three times per point and calculated the average amplitude. The results are shown in table 1 . Scattering of $B$ is larger than that of $\mathrm{A}$ and the standard deviation of $\mathrm{B}$ is 4.6 times that of $\mathrm{A}$. Consequently, the condition of the interior surface has a large effect on the amplitude of the echo.

Next, we observed echoes on the following objects, at either three or four points on each one. The measuring conditions were the same as before.

(a) A screwed round steel

(b) A screwed pipe made of round steel with boring, $3.8 \mathrm{~mm}$ thick

(c) A pipe made of round steel with boring and manufactured concentric threads having the same section as the standard cone screw and a thickness of $3.8 \mathrm{~mm}$

The pipe surfaces, both interior and exterior, were finished to $6.3 \mathrm{~s}$. Echoes are shown in figure 6-8. For (a) (shown in figure 6), only echoes propagating near the exterior surface are observed. All four data sets have similar peaks observed in

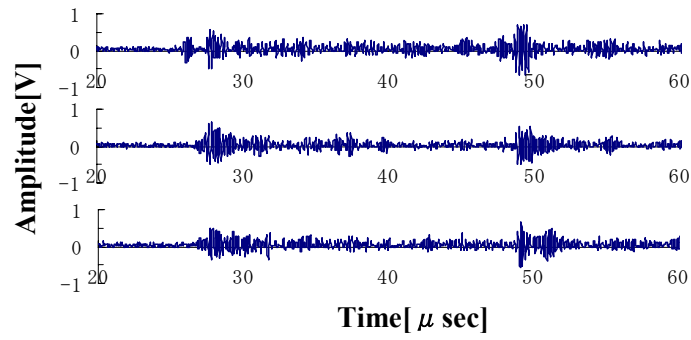

Figure 7 Wave Form on (b)

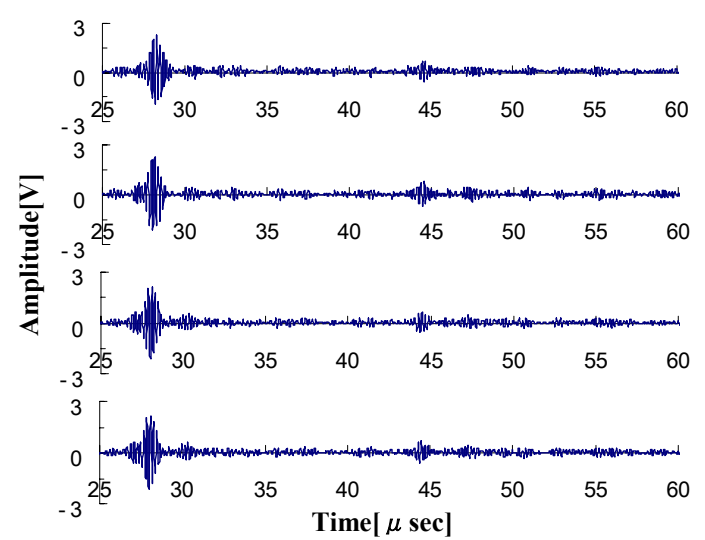

Figure 8 Wave Form on (c)

intervals of threads. But for (b) (shown in figure 7), the three data sets are different from one another. In this case, there are echoes reflecting at the interior surface. In addition, for (c) (shown in figure 8), all four data sets are very similar to one another although there are the internal reflecting waves. In this case, all axial sections are similar because the threads are concentric, not spiral. Therefore, we can consider that echoes reflected at the interior surface and the spiral of the screw cause the difference among the echoes depending on the probe position.

\section{HOW TO ESTIMATE CORROSION}

\subsection{Corrosion Size}

In this report, the objective is to detect artificial local corrosion, which is a drill hole having a flat top, manufactured inside the screwed part of a pipe. We propose a method for estimating the size of corrosion (diameter of the hole) using the fact that the amplitude of the pipe-end echo in screwed pipes changes according to the probe position by scanning in the circumferential direction.

Three kinds of artificial corrosion, 3.0, 5.0 and 7.0 $\mathrm{mm}$ in diameter, were manufactured inside the screwed pipes at a uniform depth of $1.0 \mathrm{~mm}$. 


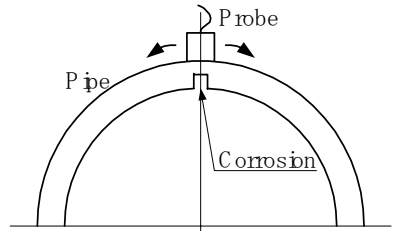

Figure 9 The way of Measuring

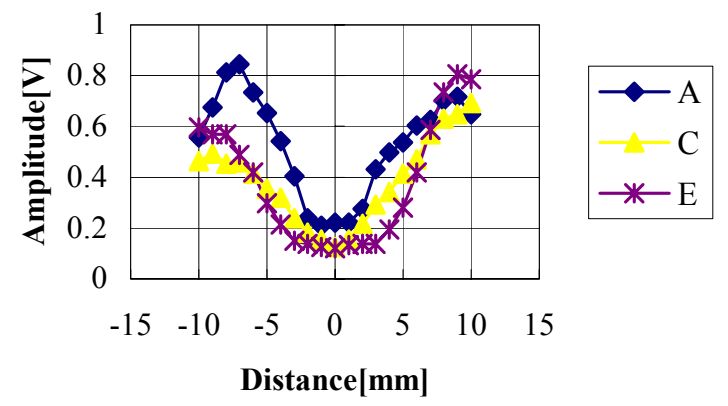

Figure 10 Pipe-End Echo amplitude (37[mm])

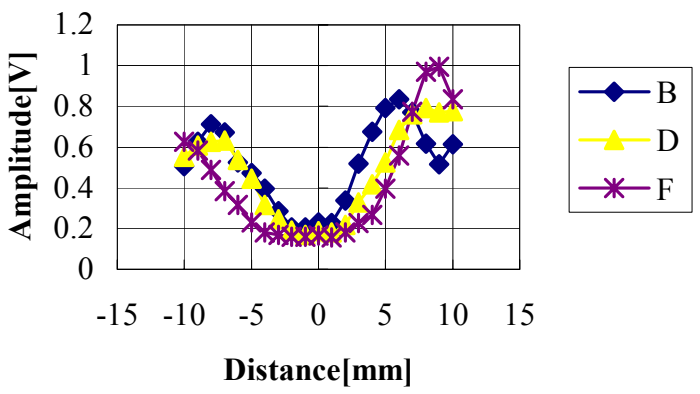

Figure 11 Pipe-End Echo amplitude (47[mm])

As shown in figure 9, we observed the echoes and measured the amplitude of the pipe-end echo with a surface SH probe. We defined the center of the corrosion as the origin for observation and moved the probe to $10 \mathrm{~mm}$ in both directions at $1.0 \mathrm{~mm}$ intervals.

There were two corrosion positions; the horizontal distance from the incidence point of the probe was $37 \mathrm{~mm}$ and $47 \mathrm{~mm}$. As table 1 indicates, the amplitude of the echo is influenced by the contact condition of the probe and the interior surface condition of the pipe, so we used three pipes for each corrosion pattern, size and position, and collected echo data three times, then calculated the average for each pipe. The diagrams positioning the average pipe-end echo amplitude are shown in figure $10(37 \mathrm{~mm})$ and figure $11(47 \mathrm{~mm})$. In these figures, "A" and " $\mathrm{B}$ " refer to corrosion having a diameter of $3 \mathrm{~mm}$, "C" and "D" refer to $5 \mathrm{~mm}$ and "E" and "F" are $7 \mathrm{~mm}$. As the diagrams indicate,
Table 2 Quadratic Coefficient

\begin{tabular}{|c|c|c|c|}
\hline Position & $\phi 3$ & $\phi 5$ & $\phi 7$ \\
\hline $37 \mathrm{~mm}$ & 0.0238 & 0.0147 & 0.0066 \\
\hline $47 \mathrm{~mm}$ & 0.0212 & 0.0131 & 0.0067 \\
\hline Average & 0.0225 & 0.0139 & 0.0067 \\
\hline
\end{tabular}

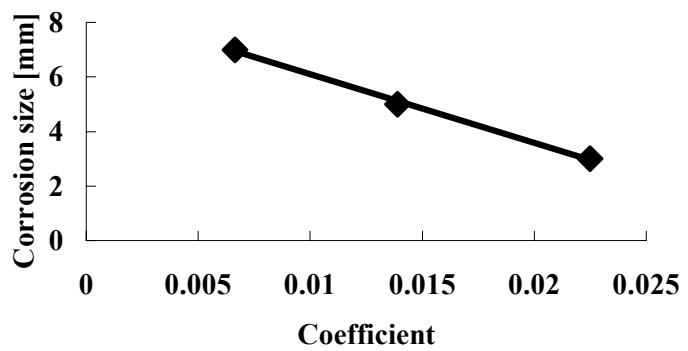

Figure 12 Regressing Line

every curved line is like a parabola. Therefore, they were approximated by a quadratic equation and each quadratic coefficient was obtained (see table 2 ) in order to estimate the size of the corrosion. The extent to which pipe-end echo is not observed spreads as the diameter of corrosion increases; accordingly the quadratic coefficient of the approximated curve becomes larger as the corrosion size decreases. Here, the number of data used for approximation differs depending on the corrosion size; 7 points were used in $\phi 3 \mathrm{~mm}, 9$ points in $\phi 5 \mathrm{~mm}$ and 11 points in $\phi 7 \mathrm{~mm}$. For each corrosion size, averages of coefficients were calculated (see table 2) and proofread using the method of least squares. It is possible to estimate the diameter of corrosion by using this regression line (see figure 12).

\subsection{Corrosion Position}

Here, position means the horizontal distance between the corrosion and the probe. It can be seen that the echo data shows a common tendency for the amplitude to decrease as time passes, that is, as the distance to the reflecting source increases, in spite of differences between the observed points. Although it is very difficult to make a completely reliable numerical model, it is possible to make a simple numerical model by using only the changes in the echo amplitude. If corrosion is on the propagation pass, it can be assumed that the amplitude would change markedly at the time for that position. Therefore, it is reasonable to assume that the position can be estimated by detecting the time. We made a simple numerical model of the 
echo amplitude and estimated the corrosion position by comparing it to actual envelope data.

\subsubsection{Modeling of Echo Amplitude}

The amplitude of echo is a function of various factors. Four of the factors, directivity, sound field, damping and reflectance, are variables. Here, we supposed that the only reflecting source is the bottom of the thread and that it is only the propagation pass that reflects on the interior surface of the pipe and the bottom of the thread, also assuming that the reflectance is constant. Directivity of this probe was measured as shown in figure 2. The sound field was expressed as a numerical model by Freedman, ${ }^{[9]}$ therefore, we used his expression. The damping coefficient was measured experimentally. As shown figure 13, we made a piece of round steel that had a horizontal drill hole $(\phi 1)$, measured the amplitude of the hole echo as changing distance $\mathrm{x}$ and calculated the damping coefficient after revising the sound field and reflectance.

The simple amplitude model of echo is shown in figure 14. Here, the horizontal axis means the distance from the incident point.

\subsubsection{Method of Estimation}

The point at which the pipe-end echo amplitude is the smallest of all points in the circumference measuring was the center of the corrosion. For the simple amplitude model, it is necessary to add an offset value because amplitude can change due to contact conditions and other factors. Therefore, the

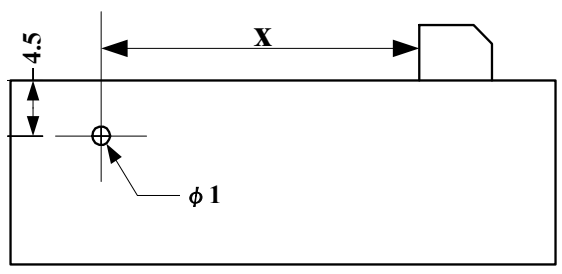

Figure 13 Measurement of Damping Coefficient

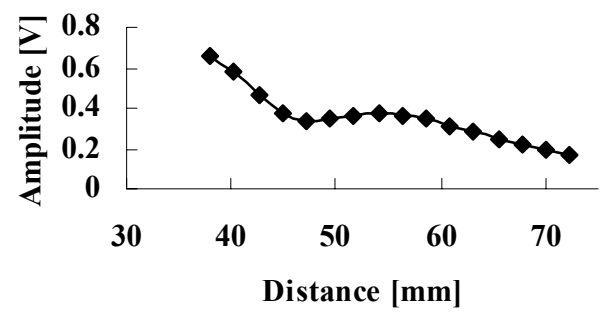

Figure 14 Simple Amplitude Model offset is calculated by comparing the simple amplitude model in figure 14 to the envelope data of the echo observed at the center of the corrosion. After that, the error between the envelope and model data is calculated, and the time for the corrosion position is detected as the one having the largest positive error.

\section{EXPERIMENT AND CONSIDERATION}

We conducted an experiment using the method described above. The object was an artificial corrosion pipe manufactured from standardized carbon steel pipe. The conditions of the experiment are shown in figure 5. Echoes were observed when moving the probe from the center of the corrosion $10 \mathrm{~mm}$ in both directions at 1.0 $\mathrm{mm}$ intervals. The size and the position of the artificial corrosion and the error of estimation are shown in tables 3 and 4. Here, the position means the horizontal distance between the incident point and the corrosion. Each type of corrosion was set up on the respective pipe.

In the estimation of size, the error for $\phi 4 \mathrm{~mm}$ is larger than that for $\phi 6 \mathrm{~mm}$, it is about 2 or $3 \mathrm{~mm}$. The cause of the error is the comparatively small pipe-end echo amplitude. It is clear that the changes in echo amplitude in figure 15 and 16 are smaller than those in figure 10 or 11 , which are data for proofreading; therefore, the size of corrosions is estimated larger than the real value. There is a limited ability to estimate size using this method because the amplitude varies depending on the pipe.

Therefore, it is necessary to analyze the relation between the screwed part of the pipe and the echoes in more detail. However, the method is sufficient for practical use because it is possible to safely detect and estimate corrosion by proofreading with appropriate data, having

Table 3 Estimation Results of Size

\begin{tabular}{|l|c|c|c|c|c|c|}
\hline & TF1 & TF2 & TF3 & TS1 & TS2 & TS3 \\
\hline Size & \multicolumn{3}{|c}{$\phi 4$} & \multicolumn{4}{c|}{$\phi 6$} \\
\hline Estimation & 7.09 & 7.54 & 6.00 & 7.18 & 6.92 & 7.86 \\
\hline Error & 3.09 & 3.54 & 2.00 & 1.18 & 0.92 & 1.86 \\
\hline
\end{tabular}

Table 4 Estimation Results of Position

\begin{tabular}{|l|c|c|c|c|c|c|}
\hline & TF1 & TF2 & TF3 & TS1 & TS2 & TS3 \\
\hline Position & \multicolumn{7}{|c|}{43} \\
\hline Estimation & 45.0 & 43.9 & 42.6 & 41.0 & 43.4 & 42.0 \\
\hline Error & 2.0 & 0.9 & -0.4 & -2.0 & 0.6 & -1.0 \\
\hline
\end{tabular}




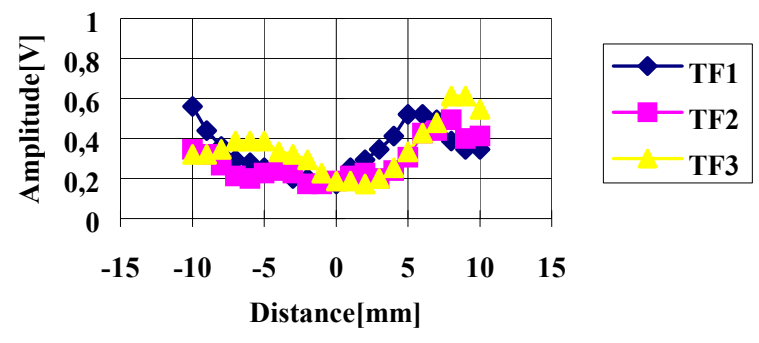

Figure 15 Pipe-End Echo Amplitude of "TF"

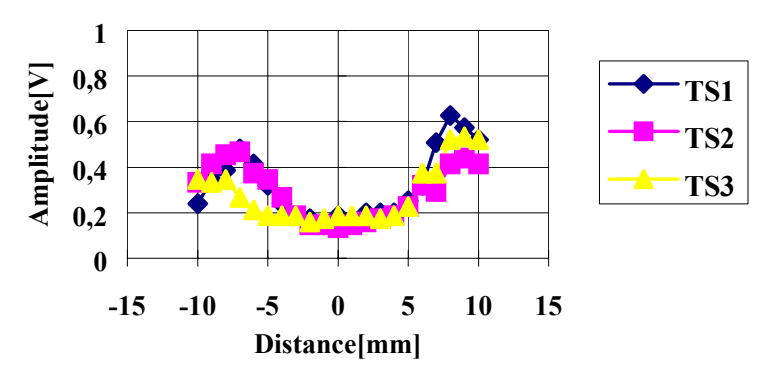

Figure 16 Pipe-End Echo Amplitude of "TS"

comparatively large changes, selected from multiple pipes.

The position can be estimated with sufficient accuracy for practical use, the error is less than \pm 2 $\mathrm{mm}$.

\section{CONCLUSION}

We attempted to estimate artificial internal corrosions of male screwed parts of pipes with a surface $\mathrm{SH}$ probe in order to conduct an inspection in air conditioning plants with ultrasonic testing. In this paper, we described the following:

(1) The results of experimental analysis on the echo from screwed parts of pipes.

(2) The method used to estimate the size of corrosion by using the change in pipe-end echo amplitude.

(3) The method used to estimate the position of corrosion by comparing the envelope measuring data to the simple numerical echo amplitude model.

Using this method, we were able to detect local corrosion with certainty and estimate the position with sufficient accuracy for practical use. However, it is necessary to analyze the relation between screwed parts of pipes and the echoes in more detail in order to improve the accuracy of estimating the size of local corrosion.

\section{REFERENCES}

[1]Kurosaki and Nakayama, "Automatic Inspection System for Piping Corrosion", Proceedings of The 1st Symposium on Construction Robotics in Japan, pp.379-384(1990)

[2]Mukai, et al, "Automatic Inspection System for Piping Corrosion (Part 1. R\&D Concept and System Outline", Technical Papers of Annual Meeting of The Society of Heating, Air-Conditioning and Sanitary Engineering of Japan, pp.933-936(1989)

[3]Sakamoto, et al, "Control of Parallel-Link Robot Manipulator and Pipe-Flaw Diagnosis", Proceeding of The 7th Symposium on Construction Robotics in Japan, pp.307-316(1998)

[4]Fukuda, et al, "In-Pipe Corrosion Diagnosis System (Estimation of the Type and Size of the Corrosion Using Two-Dimensional Ultrasonic Flaw Detector), Transaction of the Japan Society of Mechanical Engineers, Vol.68, No.666, C, pp.391-397(2002)

[5]Light, et al, "Stud bolt inspection using ultrasonic cylindrically guided wave technique", ASME ,Vol.26, pp.31-38

(1986)

[6]Bossi, et al, "Automated detection and sizing of defects in complex geometries.", The $11^{\text {th }}$ World Conference on Nondestructive Testing, pp.1540-1547 (1985)

[7]Takahashi, et al, "Experimental Study of Echo Directivity for Surface SH Wave and SH Wave Angle Probes", The $7^{\text {th }}$ European Conference on Non-Destructive Testing, pp.26-29 (1998)

[8]Kasahara, et al, "Study on the Ultrasonic Testing Techniques Using Grazing SH-Wave", Proceedings of the 3rd Symposium on Ultrasonic Nondestructive Characterization, pp.129-135 (1996)

[9]Freedman, "Sound Field of a Rectangular Piston", The Journal of Acoustical Society of America, Vol.32, No.2, pp.197-209 (1960) 